\title{
TILE: SYMMETRY BREAKING IN A QUANTUM DOUBLE-WELL CHAIN
}

\author{
AUTHOR(S): J. E. Gubernatis \\ D. K. Campbell \\ Xidi Wang
}

\section{sUaumtred To: Proceedings, Yukawa Memorial Symposium, Nishinomiy, Japan, October 24-25, 1991; Published by Plenum Press \\ DISCLAIMER}

\begin{abstract}
This report was prepared as an account of work sponsored by an agency of the United States Government. Neither the United States Government nor any agency thereof, nor any of their employees, makes any warranty, express or implied, or assumes any legal liability or responsibility for the accuracy, completeness, or usefulness of any information, apparatus, product, or process disclosed, or represents that its use would not infringe privately owned rights. Reference herein to any specific commercial product, process, or service by trade name, trademark, manufacturer, or otherwise does not necessarily constitute or imply its endorsement, recommendation, or favoring by the United States Government or any agency thereof. The views and opinions of authors expressed herein do not necessarily state or refiect those of the United States Government or any agency thereof.
\end{abstract}

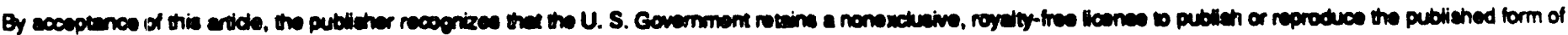
this convibution, or to sllow chere to do eo, lor U.. S. Governirent puspoese.

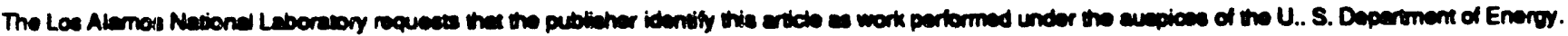

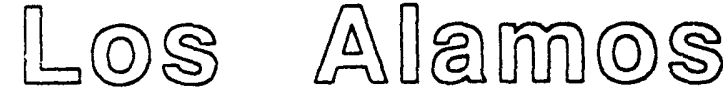

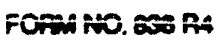
ST.NO. 20005101

\section{Los Alamos National Laboratory Los Alamos, New Mexico 87545}


Symmetry Breaking in a Quantum Double-Well Chain

J. E. Gubernatis, Theoretical Division

Los Alamos National Laboratory, Los Alamos, NM 87545, U.S.A.

D. K. Campbell and Xidi Wang, Center for Nonlinear Studies

Los Alamos National Laboratory, Los Alamos, NM 87545, U.S.A.

\begin{abstract}
We present numerical evidence that quantum fluctuations can produce a symmetric ground-state in the double-well chain, restoring the symmetry that is broken classically. In particular, we present the phase diagram for this model that shows the symmetry restoration occurs more easily than predicted by a perturbation theory calculation of the continuum limit of the model.
\end{abstract}

\title{
1. Introduction
}

The double-well chain chain is a system of particles that move on a lattice of symmetric, double-well potentials which are coupled harmonically to one another. The physicsil properties of this system are invariant to changing the sign of the displacement variables, and this symmetry leads to a doubly-degenerate ground-state. Classically, this symmetry is always broken, and the particles all sit in the left- or the right-hand side of their wells. Quantum mechanically, however, we find a range of model parameters for which symmetry is restored by quantum fluctuations.

Our interest in this model was motivated by a series of quantum Monte Carlo simulations we are performing on one-dimensional, electron-phonon models of conducting polymers. Several years ago, for similar models, Fradkin and Hirsch [1] investigated how the electron motion can generate an effective doublewell potential for the phonons and thereby cause the lattice to dimerize. They also argued that for certain models quantum fluctuations at low temperatures restore symmetry (i.e., destroy the dimerisation). The quantum double-well chain is a simpler problem on which to study similar issues and to test new numerical methods.

The model, however, is also interesting on its own. It is a discretized version of a $1+1$ dimensional quantum $\phi^{4}$ field theory. In the continuum version of the model, kink/anti-kink pairs are the elementary excitations above the ground state [2]. One of our objectives was to learn more about the nature 
and consequence of these excitations.

The model is also relevant to the study of the structural properties of such chain-like, hydrogen-bonded materials as the hydrogen halides [3]. In many cases, the energy as a function of hydrogen position has been obtained by electronic structure calculations and fitted to the physical parameters of the double-well chain. Thus, with the phase diagram of the model, we can suggest whether these materials exist in the broken symmetry phase.

\section{Model Hamiltonian}

The Hamiltonian we are considering is

$$
\hat{H}=\sum_{n=1}^{N}\left[\frac{\hat{\pi}_{n}^{2}}{2 m}+\frac{w}{2}\left(\hat{\phi}_{n}-\hat{\phi}_{n+1}\right)^{2}-\frac{k}{2} \hat{\phi}_{n}^{2}+\frac{g}{4} \hat{\phi}_{n}^{4}\right]
$$

where $\hat{\phi}_{n}$ and $\hat{\pi}_{n}$ are the coordinate and momentum operators of a particle with mass $m$ on $n^{\text {in }}$ site of a chain of length $N$. The last two terms on the right-hand side of (1) are those for the symmetric, on-site double-well potentials. These potentials have two absolute minima displaced $\pm \sqrt{k / g}$ relative to each lattice site and have an energy barrier $E_{b}=k^{2} / 4 g$ between these minima. Using the frequency $\omega=\sqrt{w / m}$, the energy $\hbar \omega$, and the displaced distance of minima to scale time, energies, and lengths, we rewrite (1) in the following dimerisionless form

$$
\hat{H}=\epsilon \sum_{n=1}^{N}\left[\frac{\gamma}{2} \hat{\pi}_{n}^{2}+\frac{\gamma}{2}\left(\hat{\phi}_{n}-\hat{\phi}_{n+1}\right)^{2}+\frac{1}{4}\left(\hat{\phi}_{n}^{4}-1\right)^{2}\right]
$$

where $\epsilon=4 E_{b} / \hbar w$ and $\gamma=w / k$, and a constant term has been added.

We will calculate the ground-state properties of (2) by doing quantum Monte Caslo simulations at successively lower and lower temperatures. The formalism on which the simulations are based requires the partition function $Z$ of the model in terms of the Feynman path integral $[4,5]$. In general, $Z=$ $\int \mathcal{D} \phi e^{-S\{\phi\}}$ where $S\{\phi\}$ is the action associated with the scalar field $\phi$. In terms of (2), we find that

$$
S\{\phi\}=\epsilon \sum_{n} \int_{0}^{\rho} d \tau\left[\frac{\gamma}{2}\left(\frac{\partial \phi_{n}}{\partial \tau}\right)^{2}+\frac{\gamma}{2}\left(\phi_{n}-\phi_{n+1}\right)^{2}+\frac{1}{4}\left(\phi_{n}^{2}-1\right)^{2}\right]
$$

where $\tau$ is the imaginary-time variable and $\beta$ is the inverse of the temperature $T$.

An implicit parameter in ( 1 ) is the lattice constant a. Letting a vanish, we can obtain the continuum version of the model. If treated classically, this 
version of the model has in the ground state $\phi= \pm 1$, an energy $E_{g}=0$, and soliton solutions representing excitations from the ground state. If ireated quantum mechanically, its ground state properties are renormalized, and the lowest order correction (at the one-loop level) [2] leads to

$$
E_{g}=\frac{2 \sqrt{2}}{3} \epsilon \sqrt{\gamma}+\frac{1}{\sqrt{\gamma}}\left(\frac{-3}{\pi \sqrt{2}}+\frac{1}{2 \sqrt{2}}\right) \text {. }
$$

When

$$
\epsilon<\frac{18-\sqrt{3} \pi}{8 \pi \gamma} \simeq \frac{0.5}{\gamma}
$$

$E_{g}$ becomes negative, then the broken symmetry ground state is unstable to the formation of kink/anti-kink pairs. This instability is an indication of the restoration of symmetry. We will find that the above estimate, while not very useful quantitatively, does point to the correct physical picture.

\section{Numerical Methods}

For the simulations, we discretize the integration in imaginary time into $L$ stepe of size $\Delta$ defined by $L \Delta=\beta$ and expreas the action $S\{\phi\}$ in terms of variable $\phi_{i, j}$

$$
S\{\phi\}=\Delta \epsilon \sum_{i, j}^{L, N}\left[\frac{\gamma}{2}\left(\frac{\phi_{i+1, j}-\phi_{i, j}}{\Delta}\right)^{2}+\frac{\gamma}{2}\left(\phi_{i, j}-\phi_{i, j+1}\right)^{2}+\frac{1}{4}\left(\phi_{i, j}^{4}-1\right)^{2}\right]
$$

Here, the subacripts $i$ and $j$ are the labels for the imaginary time and space dimensions. Formally, this action is similar to the discrete, classical, two dimensional $\phi^{4}$ system $[6,7]$. In the present case, however, to inaure we are describing quantum behavior, we have to require $\Delta$ to be much smaller than the reciprocal of $\boldsymbol{\gamma}$.

The conventional, path integral Monte Carlo procedure evaluates the expectation value of a physical quantity $A=A\{\phi\},\langle A\rangle=\int \mathcal{D} \phi A\{\phi\} e^{-\phi S\{\phi\} / Z}$ by generating a sequence $\phi_{i}$ of independent configuration of the $\phi$-fields with weight $e^{-\rho S} / Z$. The procedure reduces the computation of the expectation values to the simple summation, $(A)=\sum_{i=1}^{M} A\left\{\phi_{i}\right\} / M$ where $M$ is the number of independent configurations generated.

Instead of using the conventional Path Integral Monte Carlo method to generate the $\phi$ fields, we used the Hybrid Monte Carlo Method [8]. This method combines molecular dynamics and Monte Carlo methods. One consequence is more rapid equilibriation of the syeitem and leas correlation between succesaive measurements. Another feature is the high inherent degree of vectorization and 
hence parallelization. The combination of these features allowed us to simulate efficiently long chains at low temperatures.

To study whether the symmetry of the ground state is broken, we adopted the following strategy: for successively lower values of the temperature $T$, we study the behavior of the order parameter, the energy, and their mean-squared fluctuations as a function of $\epsilon$ and $\gamma$. In terms of the discretized action (6), we define a "quasi" inverse temperature $\beta_{q} \equiv \epsilon$ and a "quasi" (classical) twodimensional Hamiltonian

$$
K_{q} \equiv \sum_{i, j}\left[\frac{\gamma}{2 \Delta}\left(\phi_{i+1, j}-\phi_{i, j}\right)^{2}+\frac{\Delta \gamma}{2}\left(\phi_{i, j+1}-\phi_{i, j}\right)^{2}+\frac{\Delta}{4}\left(\phi_{i, j}^{2}-1\right)^{2}\right]
$$

such that $\beta_{q} H_{q}\{\phi\}=S\{\phi\}$. At each value of the physical temperature, we fix $\gamma$ and then use standard methods to determine whether the system defined by $H$, undergoes a transition from the broken to the restored symmetry state at some critical value of $\beta_{q}, \beta_{q c} \equiv \epsilon_{c}$.

Within this strategy, finding the condition for the broken symmetry at a fixed physical temperature for our one-dimensional quantum model is equivalent to finding the critical inverse quasi-temperature $\epsilon_{\mathrm{e}}$ for the two-dimensional Hamiltonian $H_{q}$. In the absence of infinite-ranged interactions, however, a true phase transition in one-dimension can only occur for an infinite-sized system at zero temperature (i.e., for $N$ and $L \rightarrow \infty$ ). Within our strategy, we search for a phase transition in two-dimensional system whose inverse temperature is $\epsilon$. Again, a true transition will only occur in an infinite system, but it can occur at a finite value of inverse quasi-temperature $\epsilon$. We seek to determine if such a transition is indicated and if these indications remain as we increase $L$ and $N$. What distinguishes our quantum simulations from those for the classical system $H_{q}$ is the need to require that the physical quantities we compute to be independent of $\Delta$ to within the accuracy of our calculation.

\section{Result:}

As one indicator of the symmetry state of the system, we took for the order parameter

$$
X(\epsilon)=\frac{1}{L N}\left\langle\left|\sum_{i, j}^{L, N} \phi_{i, j}\right|\right\rangle .
$$

As the size of the system $N$ becomes very large and the true temperature $T=1 / \Delta L$ approaches sero, the value of the order paremeter will change from a finite positive value to sero if quantum fluctuations are restoring the symmetry. We also studied the susceptibility associated with the order parameter, the energy, and the specific heat. 


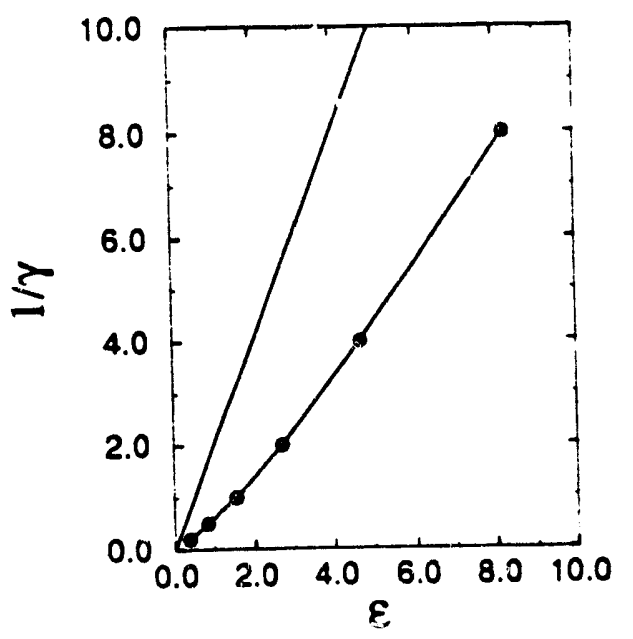

Fig. 1. The phase diagram. The straight line is the phase boundary predicted from the perturbation analysis of the continuum limit; the makers are the results obtained from the simulations. The restored symmetry phase lies above the curve represented by these markers.

We simulated a variety of system sizes, ranging from $N=7(\beta=4.95)$ to $N=128(\beta=45.25)$. The $N=128$ calculations were done on a Thinking Machincs CM-2 computer. The remainder were done on a Sun Sparcstation 1, a Convrax 230, and a Cray X-MP. The simulations on the CM-2 took about 65 minutes of computation tirne for 40,000 measurements and about 120 minutes for 80,000 . This was at least 10 times the speed of the X-MP.

To estimate $\epsilon_{c}$, we used the Cumulant Intersection Method [6, 9]. To reduce the computation time near the critical point, we used the Histogram Method of Ferrenberg and Swendsen [10] to help estimate the moments of $\phi$ needed in the cumulant method.

Our principal result is shown in Fig. 1 where we plot the phase diagram as a function of the model parameters $e$ and $1 / \gamma$. The straight line with a slope of approximately 2 is the phase boundary eatimated from the continuum theory reaults of (4) and (5). It predicts the existence of a restored symmetry phase above this line. The markers in this figure are the results from the quantum Monte Carlo simulation. Above the curve represented by these points lies the restored symmetry phase. We see that the continuum theory qualitati ely predicts the correct physics, but quantitatively the the transition actually occurs more casily.

\section{Concluding Remarks}

We have presented numerical evidence that quantum fluctuations can produce a 
symmetric ground-state in the double-well chain, restoring the symmetry that is broken classically. In particular, we presented the phase diagram for this model that shows the symmetry restoration occurs more easily than predicted by a perturbation theory calculation of the continuum limit of the model. In another paper will report the full details of our analysis and results [11].

We are currently trying to determine the universality class for the model by conformal-charge methods $[12,13]$. The implications of our results the hydrogen-halide materials will be discussed elsewhere [13].

\section{Acknowledgments}

We thank W. R. Somsky and H. Q. Lin for helpful conversations. The work was supported by the U. S. Department of Energy. We also thank the Advanced Computing Laboratory at the Los Alamos National Laboratory for the use of its facilities.

\section{References}

1. E. Fradkin and J. E. Hirsch, Phys. Rev B 27, 1680 (1982); J. E. Hirsci and E. Fradkin, Phys. Rev. B 27, 4032 (1983).

2. R. Dashen, B. Hasslacher, and A. Neveu, Phy. Rev. D 10, 4114, 4139 (1974).

3. For example, R. W. Jansen, R. Bertoneini, D. A. Pinnick, A. I. Katz, R. C. Hanson, O. F. Sankey and M. O'Keeffe, Phys. Rev. B 35, 9830 (1987).

4. R. P. Feynman and A. R. Hibbs, Quantum Mechanics and Path Integrals (McGraw-Hill, New York, 1965).

5. M. Creuts and B. Freedman, Ann. Phys. 132, 427 (1981).

6. A. Milchev, D. W. Heermann and K. Binder, J. Stat. Phys. 44,749 (1986).

7. R. Toral sad A. Chakrabari, Phys. Rev. B 42, 2445 (1990).

8. S. Duane, A. D. Kennedy, B. J. Pendelton, and D. Roweth, Phys. Lett. B 195, 216 (1987).

9. K. Binder, in Applications of the Monte Carlo Method to Statistical Physics, edited by K. Binder (Springer-Verlag, Berlin, 1984), Chap. 1.

10. A. M. Ferrenberg and R. Swendsen, Phys. Rev. Lett. 61, 2635 (1988); Phys. Rev. Lett. 63, 1195 (1989).

11. Xidi Wang, D. K. Campbell, J. E. Gubernatis, "Symmmetry breaking in a quantum double-well chain, ${ }^{n}$ unpublished.

12. Rajiv R. P. Singh and G. A. Baker, Jr., Phys. Rev. Lett. 61, 1 (1991).

13. Xidi Wang, D. K. Campbell, G. A. Baker, Jr., and J. E. Gubernatis, "Conformal charge of the two-dimensional $\phi^{4}$ field theory," unpublished. 

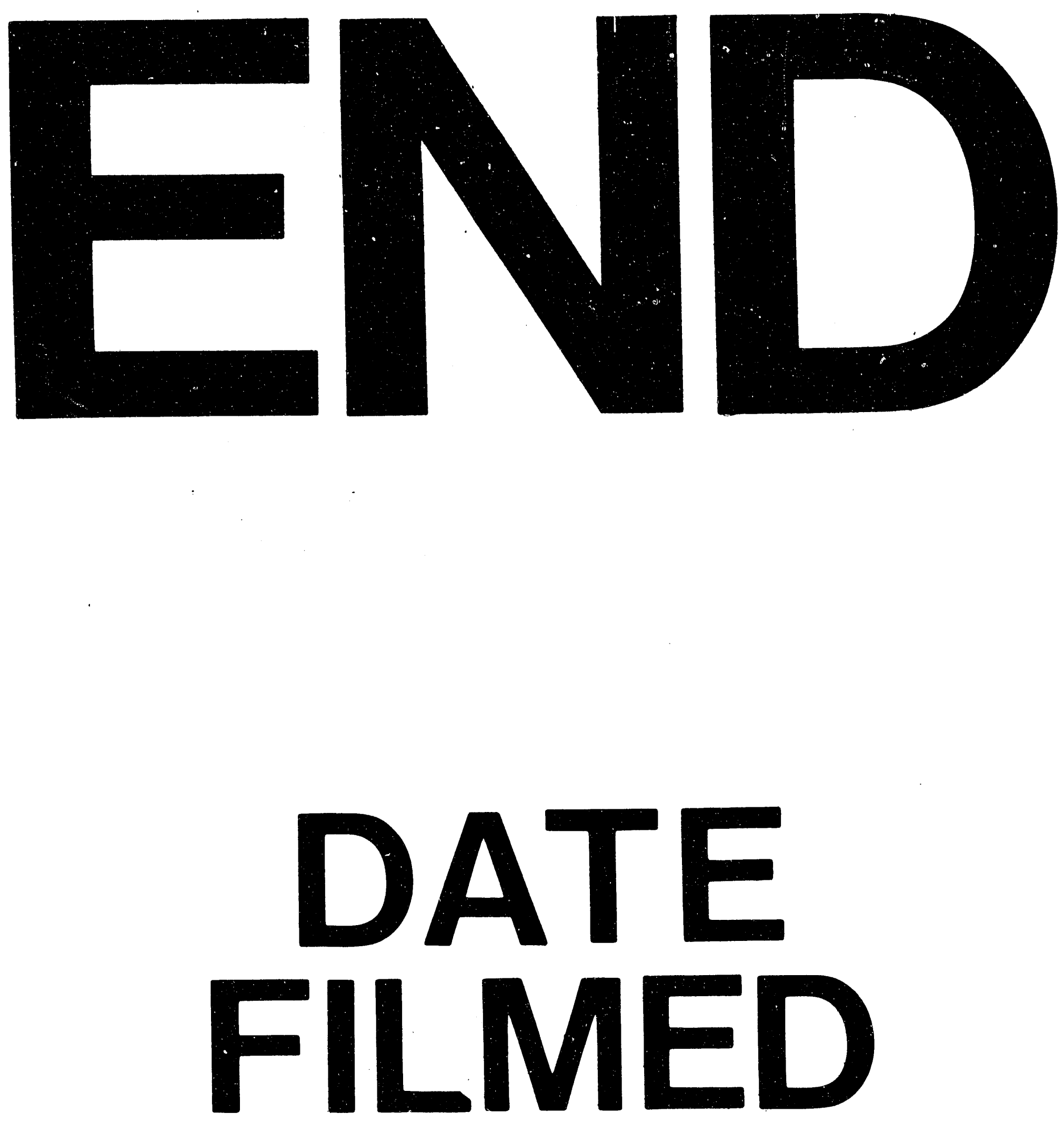

1

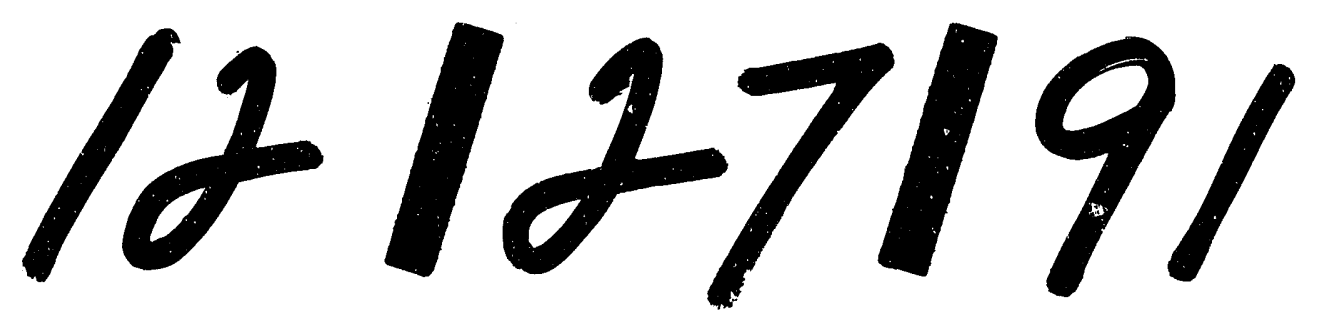


